\title{
Routine use of antifungals in cancer treatment questioned
}

\section{Gotzsche $P C$, Johansen $H K$. Routine versus selective antifungal administration for control of fungal infections in patients with cancer (Cochrane Review). In. The Cochrane Library, Issue 2, 1999, Oxford: Update Software.}

Objective To study in cancer patients with neutropenia whether antifungal agents given prophylactically or empirically decrease morbidity and mortality.

Data sources Medline search 1996 to September 1997, using a comprehensive search strategy. Unpublished trials from conference proceedings and industry contact.

Study selection Randomised trails comparing amphotericinB, AmBisome, fluconazole, ketoconazole, miconazole or itraconazole with placebo or no treatment.

Results 25 trials comprising 2912 randomised patients were included of whom 444 died. Prophylactic or empiric treatment with antifungals had no effect on mortality (odds ratio 0.92 [0.75-1.14]). Amphotericin decreased mortality significantly (OR $0.58[0.37-0.93])$ but the studies were small and the difference in the number of deaths was only 15 . Antifungal treatment decreased the incidence of invasive fungal infection significantly (OR 0.49 [0.36-0.66]) and also decreased fungal colonisation and use of additional (escape) antifungal therapy. The NNT to prevent one case of fungal invasion to occur in surviving patients is 59 ( CI 37-131).

Conclusions There was no convincing effect on mortality and only a modest effect on fungal invasion. We therefore question the current widespread practices of routine prophylactic or empirical therapy with antifungal agents on cancer patients with neutropenia and suggest that these agents are restricted to patients with proven infection and to patients in randomised controlled trials. A large, definitive, placebo controlled trial of amphotericin B should be performed.

Address for reprints: Peter Gotzsche The Nordic Cochrane Centre

Righospitalet, dept. 711218B Tagensvej DK-2200 Copenhagen,

Denmark

\section{Commentary}

Treatment for cancer often includes the prophylactic use of antifungal agents in an attempt to prevent morbidity or mortality from fungal infection. This review by Gotzsche and Johansen is a Cochrane systematic review that not only investigates this treatment philosophy but also comments on fundamental clinical and methodological issues.

The title of the review implies routine versus selective antifungal agents are being compared. However, the objective clarifies that both prophylactic and empirical agents are being reviewed. Whilst the objective defines the population as cancer patients with neutropenia no information is given about the white cell counts.

The methodology of the review is according to Cochrane protocol and the two reviewers worked independently. The search strategy is explicit and the electronic databases searched were MEDLINE and Cochrane. It is unclear how much hand searching has been done in this field, however, great lengths were taken to include grey literature and personal communications altered some published data. The criteria for decisions for data extraction are clear and the studies are well summarised.

Information on the quality of included studies is given and the results of a sensitivity analysis reported. Inclusion of data from 3 studies reporting empirical use of antifungal agents confuses the explicit issue of prophylactic agents. A subgroup analysis of empirical use was performed, however, this was not reported for prophylactic agents only. We feel that clinical interpretation would have been clearer if the analysis had been restricted to prophylactic agents.

In the discussion the authors raise important issues associated with both interpretation of clinical data from studies conducted on such severely ill patients and the process of conducting reviews. For example, the authors decided to use total mortality rather than mortality due to fungal infection to avoid bias and this will include possible adverse drug effects. Contacts with medical companies were not successful.
This demonstrates the need for change. Patients willingly contribute to clinical trials for the benefit of future patients and therefore the information should be regarded as public property for the public good.

The results are combined for all therapeutic agents and presented for death, invasive infection, colonisation and use of escape drugs.

The conclusion questions the current practice of using routine prophylactic or empirical therapy with antifungal agents in cancer patients. We agree with this if death is the outcome of interest, but query this for the reduction in fungal invasion. From the patient's point of view the modest reduction in fungal invasion found may be of great importance to their comfort. We agree with the authors' research recommendation that a large definitive clinical trial of amphotericin B should be carried out.

\section{Dr Janet Clarkson}

University of Dundee, $U K$.

Dr Helen Worthington

University of Manchester, $U K$. 